\title{
Pulmonary lesion subtypes recognition of COVID-19 from radiomics data with three-dimensional texture characterization in computed tomography images
}

\author{
Wei Li ${ }^{1}$, Yangyong Cao ${ }^{2 *}$, Kun Yu ${ }^{3}$, Yibo Cai ${ }^{2}$, Feng Huang ${ }^{4}$, Minglei Yang ${ }^{4}$ and Weidong $\mathrm{Xie}^{2^{*}}$ (i)
}

\author{
${ }^{*}$ Correspondence: \\ 1971582@stu.neu.edu.cn; \\ 1910638@stu.neu.edu.cn \\ ${ }^{2}$ School of Computer \\ Science and Engineering, \\ Northeastern University, \\ Shenyang, China \\ Full list of author information \\ is available at the end of the \\ article
}

\begin{abstract}
Background: The COVID-19 disease is putting unprecedented pressure on the global healthcare system. The CT (computed tomography) examination as a auxiliary confirmed diagnostic method can help clinicians quickly detect lesions locations of COVID19 once screening by PCR test. Furthermore, the lesion subtypes classification plays a critical role in the consequent treatment decision. Identifying the subtypes of lesions accurately can help doctors discover changes in lesions in time and better assess the severity of COVID-19.
\end{abstract}

Method: The most four typical lesion subtypes of COVID-19 are discussed in this paper, which are GGO (ground-glass opacity), cord, solid and subsolid. A computeraided diagnosis approach of lesion subtype is proposed in this paper. The radiomics data of lesions are segmented from COVID-19 patients CT images with diagnosis and lesions annotations by radiologists. Then the three-dimensional texture descriptors are applied on the volume data of lesions as well as shape and first-order features. The massive feature data are selected by HAFS (hybrid adaptive feature selection) algorithm and a classification model is trained at the same time. The classifier is used to predict lesion subtypes as side decision information for radiologists.

Results: There are 3734 lesions extracted from the dataset with 319 patients collection and then 189 radiomics features are obtained finally. The random forest classifier is trained with data augmentation that the number of different subtypes of lesions is imbalanced in initial dataset. The experimental results show that the accuracy of the four subtypes of lesions is $(93.06 \%, 96.84 \%, 99.58 \%$, and $94.30 \%)$, the recall is (95.52\%, $91.58 \%, 95.80 \%$ and $80.75 \%$ ) and the $f$-score is (93.84\%, $92.37 \%, 95.47 \%$, and $84.42 \%$ ).

Conclusion: The three-dimensional radiomics features used in this paper can better express the high-level information of COVID-19 lesions in CT slices. HAFS method aggregates the results of multiple feature selection algorithms intersects with traditional methods to filter out redundant features more accurately. After selection, the subtype of COVID-19 lesion can be judged by inputting the features into the RF (random forest) model, which can help clinicians more accurately identify the subtypes of COVID-19 lesions and provide help for further research. author(s) and the source, provide a link to the Creative Commons licence, and indicate if changes were made. The images or other third party material in this article are included in the article's Creative Commons licence, unless indicated otherwise in a credit line to the material. If material is not included in the article's Creative Commons licence and your intended use is not permitted by statutory regulation or exceeds the permitted use, you will need to obtain permission directly from the copyright holder. To view a copy of this licence, visit http:// creativecommons.org/licenses/by/4.0/. The Creative Commons Public Domain Dedication waiver (http://creativecommons.org/publi cdomain/zero/1.0/) applies to the data made available in this article, unless otherwise stated in a credit line to the data. 
Keywords: COVID-19, Lesion subtypes, 3D texture feature, Random forest, Hybrid adaptive feature selection, Radiomics

\section{Introduction}

In December 2019, the 2019 Coronavirus disease (COVID-19) began to spread worldwide [1, 2]. According to statistics from Johns Hopkins University: as of June 8, 2021, $173,533,746$ people worldwide have been diagnosed with the virus, and the death toll is 3,734,475 [3]. With the rapid growth of patients of the COVID-19, the shortage of clinicians is increasingly severe. Currently, clinicians mainly use RT-PCR (reverse transcription-polymerase chain reaction) technology to detect RNA in sputum or nasopharyngeal swabs to detect COVID-19 pneumonia. But this method has a certain false-negative rate 40\% [4]. Therefore, clinicians will also use chest CT images as a additional diagnostic method to improve the accuracy of COVID-19 detection for confirmed diagnosis. Moreover, the imaging pattern can change rapidly in a short period of time within the treatment process [5]. During the early COVID-19 surgery, Peng et al. [6] found that the COVID-19 lesions in CT had different subtypes, including GGO, cord, solid and subsolid. Zhang et al. [7] believe that different COVID-19 lesion subtypes have their own unique features.

However, the existing work mainly focuses on lesion detection of COVID-19 or its severity assessment. Few studies are paid attention to the classification of lesion subtypes, which ignores the important role of lesion subtypes in the diagnosis of COVID19 disease. The subtypes identification of lesions in a timely manner can enable clinicians to better assess the patient's condition and prescribe precise medicines in personality. Zhao et al. $[8,9]$ pointed out the severity and the symptoms of COVID19 pneumonia are different from common pneumonia, and lesions and characteristics of it are also different from common pneumonia. So the lesions caused by COVID-19 pneumonia are more worthy of further study. At the same time, if the patient lesion type can be determined more accurately, the doctor can more accurately determine the COVID-19 patient's condition by referring to the lesion type. In addition, training deep convolutional neural networks to diagnose COVID-19 requires massive CT impression data and huge computing resources, otherwise it is easy to cause underfitting of the model. As we all know, it is difficult to collect so much real CT images of COVID-19 for experimentation in a short period of time, while the machine learning algorithm can ignore the shortcomings of small amount of data and insufficient computing resources. Moreover, the machine learning methods of classification for COVID-19 are mainly based on features extracting from the 2D CT images in the exist papers.

To address above issues, we propose a novel random forest-based on hybrid adaptive feature selection (HAFS-RF) for CT slices. Thus, HAFS-RF first extracts the features of the lesion based on the CT slices, and filters redundant features by HAFS. Finally, the subtype of the lesion is judged according to the retained characteristics. It is worth mentioning that 3D radiomics features we used can make full use of the advanced information of lesions which is not discussed in present work to the best of our knowledge in the state of art. Therefore, HAFS-RF helps to study the computer-aided diagnosis of COVID-19 lesion subtypes recognition, at the same time, the study can reduce the 
image reading burdens of radiologists in vast data. The contributions in this paper can be discussed by three aspects.

1. The pilot research work on lesions subtypes of COVID-19 is discussed in this paper, which has never been seen in previous studies so far and may greatly assist doctor diagnosis and evaluating severity of COVID-19 patients more effectively.

2. The 3D texture radiomics analysis method is applied on COVID-19 lesions diagnosis which is better to explore more hidden inner characters within the lesions to help experts better understand the pathological features of COVID-19.

3. Extensive experiments on clinical real-world datasets demonstrate the effectiveness of the proposed model of hybrid adaptive feature selection method. Moreover, we show the capability of the proposed model for the high dimension feature data with serious imbalance problem.

The rest of the paper is organized as follows. This paper first briefly summarizes the related works. We next introduce introduce the method with the composition of the dataset, the characteristics of 3D features, and the feature selection strategy in "Materials and methods" section. The experimental results on the prepared database are discussed in "Results" section. We finally conclude this paper and look forward to the future work in "Conclusion" section .

\section{Related work}

The research work of lesion segmentation has achieved good results in the diagnosis of COVID-19 through machine learning or deep learning methods.

In terms of machine learning, Shi et al. [10] use medical imaging features and clinical features as input, and logistic regression as a classifier to distinguish COVID-19. Barstugan et al. [11] have made improvements in 2D feature extraction. GLCM (gray-level co-occurrence matrix), LDP (local directional pattern), GLRLM (gray-level run length matrix), GLSZM (gray-level size zone matrix), and DWT (discrete wavelet transform) were used to obtain the second-order statistical features for classification of COVID19. Ozkaya et al. [12] also propose a new method that fuses and ranks deep features for early detection in SVM. Elaziz et al. [13] used the new fractional multi-channel exponent moments (FrMEMs) to extract features from chest X-ray images. Then an improved Manta-ray search optimization based on differential evolution is used to select the most important features and a K-nearest neighbor classifier is used to distinguish COVID-19. Tuncer et al. [14] proposed a feature generation method called Residual Exemplar Local Binary Pattern (ResExLBP) and used a novel Iterative ReliefF (IRF) for feature selection. In their work, the SVM classifier achieved $100.0 \%$ classification accuracy by using tenfold cross-validation.

In addition, some scholars have proposed some deep learning methods for the diagnosis of COVID-19. Zhou et al. [15] segment COVID-19 lesions from CT by using the U-Net segmentation network with a spatial and multi-channel attention mechanism to assist in diagnosis COVID-19. Khan et al. [16] proposed a deep convolutional neural network called Coro-Net based on Xception architecture, which can detect COVID-19 infection from chest X-ray images. Afshar et al. [17] pointed out that CNN is easy to lose 
the spatial information between image instances, so an alternative framework based on the capsule network is proposed, which can handle small datasets. Khalifa et al. [18] finetuned deep transfer learning for limited datasets to detect pneumonia chest X-ray based on generative confrontation network. Minaee et al. [19] trained four popular convolutional neural networks, including ResNet18, ResNet50, SqueezeNet, and DenseNet-121, to identify COVID-19 disease in the analyzed chest X-ray images. He et al. [20] propose a synergistic learning framework for automated severity assessment of COVID-19 in 3D CT images, by jointly performing lung lobe segmentation and multi-instance classification. Xu et al. [21] use a 3D deep learning model to segment candidate infection areas from lung CT images, then score these areas, and finally uses noise or Bayes function to calculate the final confidence score to classify patients as COVID-19, Influenza-A viral pneumonia (IAVP), and not infected.

\section{Results}

In this section, the classifier evaluation criteria are illustrated firstly. Then, we present experimental results achieved by different methods on the evaluation dataset. Finally, the comparative experiments are conducted to prove the influence of data augmentation, 3D features and HAFS.

\section{Experimental evaluation of HAFS with RF model}

We evaluate HAFS with RF (HAFS-RF) model on the collected chest CT images dataset. Table 1 shows the quantitative results achieved by different methods.

From Table 1, we can clearly observe that HAFS-RF achieved an accuracy of (93.06\%, 96.84\%, 99.58\% and 94.3\%) for label 1, 2, 3 and 4, respectively, under the condition of $\alpha=0.5$. Followed by DT $(91.49 \%, 95.53 \%, 98.89 \%$ and $91.79 \%)$, next is RF $(90.42 \%$, 93.48\%, 98.79\% and 91.30\%). The accuracy of the remaining models such as SVM, KNN, LR, GaussianNB, and QDA is much lower than theirs. Obviously, HAFS-RF achieved the best performance. For each method, especially the accuracy of QDA is (63.72\%, $66.82 \%, 99.97 \%$ and $79.07 \%$ ), the accuracy of label 3 is always the highest, some of them even close to 100 . The possible reason for this phenomenon is that although we have enhanced the data in the experiment, the number of the four types of lesions tends to be balanced. However, the number of lesions on label 3 is still the least. On the contrary, the precision value of GaussianNB is $(88.57 \%, 42.62 \%, 14.64 \%$ and $37.82 \%)$, and the fitting ability is seriously insufficient. The reason may be that GaussianNB is prone to underfitting for a small number of samples.

Figure 1 shows the ROC curves of different models. It is also obvious that our method has the highest ROC curve area. These results all show that HAFS-RF can improve the performance and efficiency of COVID-19 classification.

\section{Influence of data augmentation}

To evaluate the effectiveness of the data augmentation, we compare it to without data augmentation, with the results reported in Table 2. The algorithm used for data enhancement is ADASYN, which is a widely used method for the data processing stage before the experiment. The main idea is: firstly, the total number of samples that need to be synthesized are calculated for minority samples. Secondly, for each minority sample, 
Table 1 Performance of COVID-19 classification achieved by SVM, KNN, LR, GaussianNB, QDA, RF, HAFS-RF $(\alpha=0.5)$

\begin{tabular}{|c|c|c|c|c|c|}
\hline Method & Label & Precision (\%) & Recall (\%) & Accuracy (\%) & F-measure (\%) \\
\hline \multirow[t]{4}{*}{ SVM } & 1 & 76.34 & 99.42 & 82.3 & 86.37 \\
\hline & 2 & 99.48 & 62.07 & $92.31 \%$ & 76.45 \\
\hline & 3 & 100.0 & 57.75 & 98.04 & 73.21 \\
\hline & 4 & 96.84 & 58.2 & 91.75 & 72.71 \\
\hline \multirow[t]{4}{*}{ KNN } & 1 & 88.04 & 86.32 & 85.66 & 87.17 \\
\hline & 2 & 83.09 & 83.23 & 93.19 & 83.16 \\
\hline & 3 & 78.05 & 86.49 & 98.17 & 82.05 \\
\hline & 4 & 65.98 & 67.96 & 87.58 & 66.96 \\
\hline \multirow[t]{4}{*}{ LR } & 1 & 83.46 & 88.4 & 83.8 & 85.86 \\
\hline & 2 & 76.93 & 75.3 & 89.83 & 76.11 \\
\hline & 3 & 66.67 & 52.11 & 96.58 & 58.5 \\
\hline & 4 & 55.86 & 50.27 & 83.7 & 52.92 \\
\hline \multirow[t]{4}{*}{ GaussianNB } & 1 & 88.57 & 59.6 & 72.88 & 71.25 \\
\hline & 2 & 42.62 & 62.72 & 75.52 & 50.75 \\
\hline & 3 & 14.64 & 86.62 & 76.01 & 25.05 \\
\hline & 4 & 37.82 & 10.19 & 79.89 & 16.05 \\
\hline \multirow[t]{4}{*}{ QDA } & 1 & 95.14 & 36.67 & 63.72 & 52.94 \\
\hline & 2 & 39.1 & 97.27 & 66.82 & 55.78 \\
\hline & 3 & 100.0 & 99.3 & 99.97 & 99.65 \\
\hline & 4 & 43.38 & 48.66 & 79.07 & 45.87 \\
\hline \multirow[t]{4}{*}{ DT } & 1 & 91.85 & 92.57 & 91.49 & 92.21 \\
\hline & 2 & 91.53 & 87.21 & 95.53 & 89.32 \\
\hline & 3 & 86.75 & 90.34 & 98.89 & 88.51 \\
\hline & 4 & 78.36 & 79.93 & 91.79 & 79.14 \\
\hline \multirow[t]{4}{*}{ RF } & 1 & 89.70 & 93.31 & 90.42 & 91.47 \\
\hline & 2 & 84.92 & 83.59 & 93.48 & 84.25 \\
\hline & 3 & 86.11 & 87.94 & 98.79 & 87.02 \\
\hline & 4 & 80.78 & 72.53 & 91.30 & 76.43 \\
\hline \multirow[t]{4}{*}{ HAFS-RF (our) } & 1 & 92.21 & 95.52 & 93.06 & 93.84 \\
\hline & 2 & 93.17 & 91.58 & 96.84 & 92.37 \\
\hline & 3 & 95.14 & 95.8 & 99.58 & 95.47 \\
\hline & 4 & 88.43 & 80.75 & 94.3 & 84.42 \\
\hline
\end{tabular}

Bold values indicate the maximum value of each type of lesion classification index

find its neighbors and calculate a weight to automatically determine the number of samples that need to be synthesized for the sample.

As can be seen from Table 2, after data augmentation, the number of the four types of lesions changed from $(2637,519,103$ and 475) to $(2637,1098,386$ and 976). The data augmentation consistently achieves better results in label 2, 3 and 4, and worse in label 1. For example, data augmentation achieves (93.84\%, 92.37\%, 95.47\% and $84.42 \%)$ in terms of $F$-measure, none achieve (93.84\%, 92.37\%, 95.47\% and $84.42 \%)$. The possible reason is that the excessive number of samples of label 1 leads to the over-fitting of the model. On the contrary, the insufficient number of other types leads to insufficient fitting ability. After using data augmentation, the four sample sizes are relatively balanced, thus avoiding over-fitting to label 1 , so the score of label 1 decreases, but the overall score increases. 


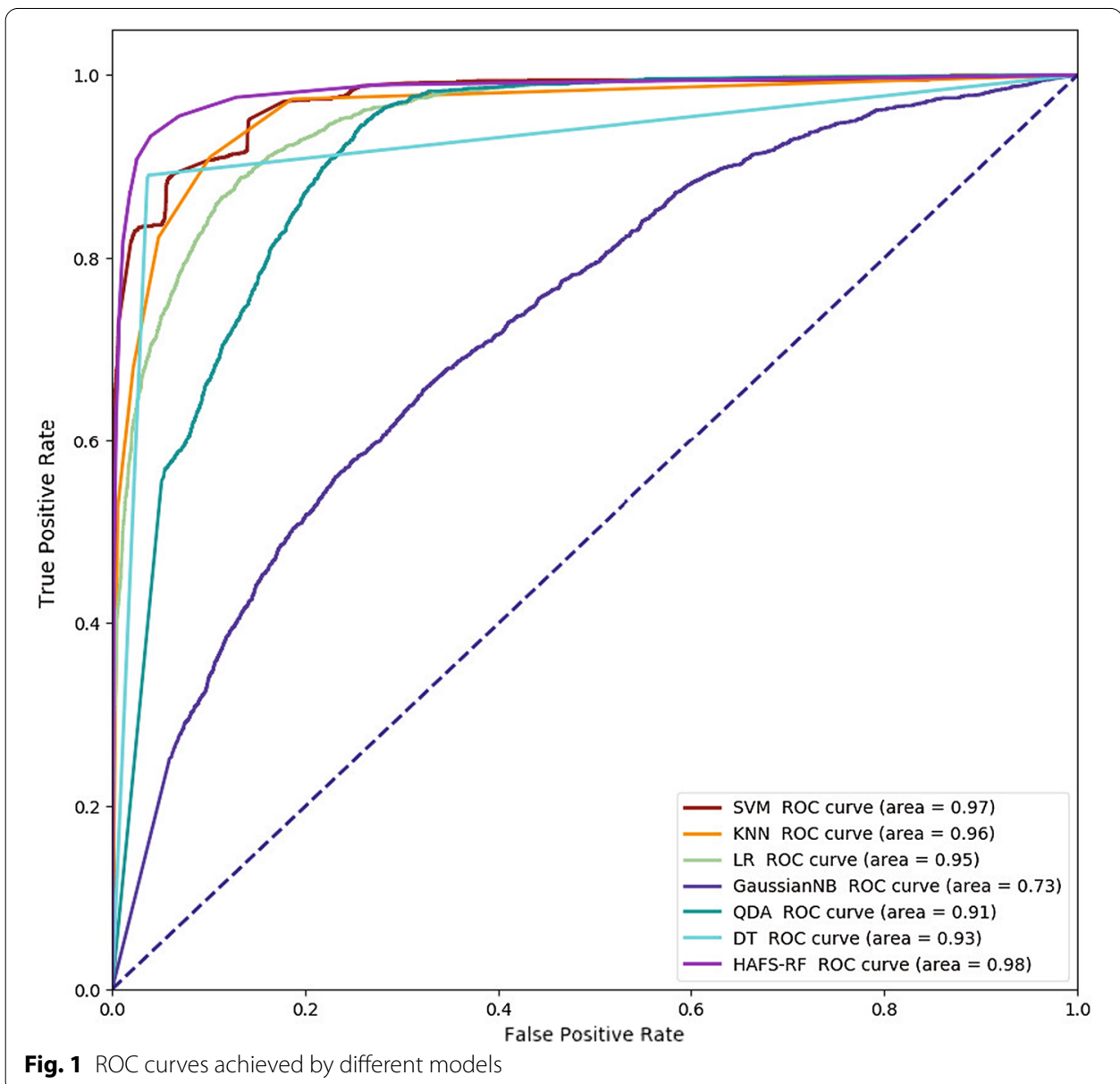

Table 2 Performance of COVID-19 classification achieved with data augmentation

\begin{tabular}{lllllll}
\hline Augmentation & Label & Number & Precision (\%) & Recall (\%) & Accuracy (\%) & F-measure (\%) \\
\hline With & 1 & 2637 & 93.17 & 96.85 & 92.95 & 94.97 \\
& 2 & 519 & 89.84 & 86.02 & 96.88 & 87.89 \\
& 3 & 103 & 89.47 & 77.27 & 99.16 & 82.93 \\
Without & 4 & 475 & 82.94 & 73.25 & 93.55 & 77.79 \\
& 1 & 2637 & 92.21 & 95.52 & 93.06 & 93.84 \\
& 2 & 1098 & 93.17 & 91.58 & 96.84 & 92.37 \\
& 3 & 386 & 95.14 & 95.8 & 99.58 & 95.47 \\
& 4 & 976 & 88.43 & 80.75 & 94.3 & 84.42 \\
\hline
\end{tabular}

\section{Influence of 3D features}

As shown in Fig. 2, each feature will have its own score in HAFS. The green features are discarded in the first stage of HAFS, the orange features are discarded in the second stage of HAFS, and the blue features are selected after HAFS.

The details of blue features of Fig.2 are shown in Table 3. It is obvious that after feature selection, a total of 48 features out of 189 features were retained. Among them, 


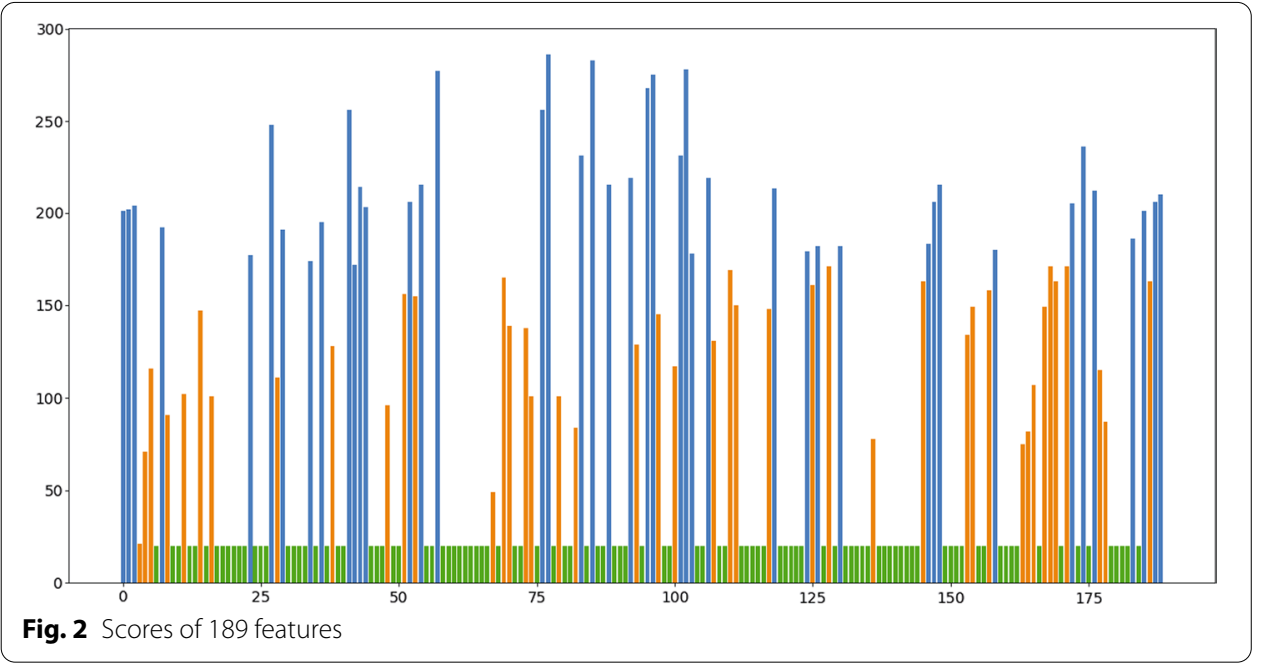

Table 3 The features after the process of HAFS

\begin{tabular}{|c|c|c|}
\hline $\begin{array}{l}\text { Dimension } \\
\text { of features }\end{array}$ & Kind of features & Characteristics \\
\hline $2 \mathrm{D}$ & First order & Length, Mean, Max, Var, ASM, Energy \\
\hline \multirow[t]{6}{*}{$3 \mathrm{D}$} & First order & $\begin{array}{l}\text { Robust mean absolute deviation, Mean, Root mean squared, Range, Interquar- } \\
\text { tile range, Skewness }\end{array}$ \\
\hline & Glszm & $\begin{array}{l}\text { Gray-level variance, High gray-level zone emphasis, Zone percentage, Small area } \\
\text { low gray-level emphasis }\end{array}$ \\
\hline & Glrlm & $\begin{array}{l}\text { Long-run high gray-level emphasis, Difference variance, Gray-level nonuniform- } \\
\text { ity normalized, Run percentage }\end{array}$ \\
\hline & $\mathrm{Glcm}$ & Sum squares, Id, Joint average \\
\hline & Gldm & $\begin{array}{l}\text { Dependence nonuniformity normalized, Dependence entropy, Dependence } \\
\text { entropy }\end{array}$ \\
\hline & Shape & Major axis length \\
\hline
\end{tabular}

there are $18(6 \times 3) 2 \mathrm{D}$ features and 30 3D features. More 3D features are retained than 2D features. So 3D features may be more effective than 2D features.

To study the influence of 3D features, a comparison was done with and without the 3D features for our model. Results of the evaluated criteria are given for the 189 features in Table 4. As shown in Table 4, HAFS-RF achieves the better classification accuracy when we use 2D and 3D features (93.06\%, 96.84\%, 99.58\% and 94.3\%) than when we use 2D features $(89.37 \%, 93.12 \%, 98.47 \%$ and $91.2 \%)$. The possible reason for improvements is that the $3 \mathrm{D}$ features we use have high-level feature representation, thereby improving the typing performance.

\section{Influence of HAFS}

To study the effectiveness of the HAFS selection, firstly we compare HAFS with stateof-the-art feature selection methods (F-test, MIC, REF, and Lasso). Since they cannot determine the optimal number of features, we select the same number of features of them as HAFS-RF $(\alpha=0.5)$ for comparison experiments. The results are reported in Table 5 . 
Table 4 Performance of COVID-19 classification achieved by using 2D features and using 2D and 3D features

\begin{tabular}{llllll}
\hline Feature & Label & Precision (\%) & Recall (\%) & Accuracy (\%) & F-measure (\%) \\
\hline 2D & 1 & 88.38 & 93.56 & 89.37 & 90.89 \\
& 2 & 84.18 & 81.9 & 93.12 & 83.02 \\
& 3 & 85.94 & 79.14 & 98.47 & 82.4 \\
\multirow{2}{*}{ 2D and 3D } & 4 & 79.84 & 69.29 & 91.2 & 74.19 \\
& 1 & 92.21 & 95.52 & 93.06 & 93.84 \\
& 2 & 93.17 & 91.58 & 96.84 & 92.37 \\
& 3 & 95.14 & 95.8 & 99.58 & 95.47 \\
& 4 & 88.43 & 80.75 & 94.3 & 84.42 \\
\hline
\end{tabular}

Table 5 Performance of different feature selection algorithm achieved by F-test, MIC, RFE, Lasso, HAFS $(\alpha=0.5)$ using Random Forest

\begin{tabular}{llllll}
\hline Method & Label & Precision (\%) & Recall (\%) & Accuracy (\%) & F-measure (\%) \\
\hline F-test & 1 & 86.59 & 91.66 & 87.32 & 89.05 \\
& 2 & 78.32 & 71.99 & 90.51 & 75.02 \\
& 3 & 74.62 & 64.67 & 97.2 & 69.29 \\
MIC & 4 & 71.43 & 67.52 & 88.66 & 69.42 \\
& 1 & 87.4 & 93.09 & 88.69 & 90.16 \\
& 2 & 76.75 & 75.04 & 90.91 & 75.89 \\
& 3 & 88.0 & 70.06 & 97.98 & 78.01 \\
RFE & 4 & 73.42 & 65.59 & 88.27 & 69.28 \\
& 1 & 84.82 & 93.32 & 86.99 & 88.87 \\
& 2 & 81.29 & 77.26 & 92.28 & 79.23 \\
& 3 & 88.07 & 61.15 & 97.59 & 72.18 \\
Lasso & 4 & 75.43 & 63.97 & 88.53 & 69.23 \\
& 1 & 87.69 & 93.44 & 89.05 & 90.47 \\
& 2 & 77.84 & 73.85 & 91.0 & 75.79 \\
& 3 & 80.45 & 68.15 & 97.52 & 73.79 \\
& 4 & 74.69 & 67.69 & 88.85 & $\mathbf{7 1 . 0 2}$ \\
HAFS & 1 & $\mathbf{9 2 . 2 1}$ & $\mathbf{9 5 . 5 2}$ & $\mathbf{9 3 . 0 6}$ & $\mathbf{9 3 . 8 4}$ \\
& 2 & $\mathbf{9 3 . 1 7}$ & $\mathbf{9 1 . 5 8}$ & $\mathbf{9 6 . 8 4}$ & $\mathbf{9 2 . 3 7}$ \\
& 3 & $\mathbf{9 5 . 1 4}$ & $\mathbf{9 5 . 8}$ & $\mathbf{9 9 . 5 8}$ & $\mathbf{9 4 . 4 7}$ \\
\hline
\end{tabular}

Bold values indicate the maximum value of each type of lesion classification index

One can observe from Table 5 that compared to the other four methods, HAFS gets the highest accuracy $(93.06 \%, 96.84 \%, 99.58 \%$ and $94.3 \%)$. This proves from the side that the features selected by HAFS are more representative.

Secondly, we further develop four methods based on SVM, KNN, GaussianNB, and QDA by using HAFS (i.e., HAFS-SVM, HAFS-KNN, HAFS-GaussianNB, and HAFSQDA). We evaluate these eight methods, with the results reported in Table 6.

As shown in Table 6, the accuracy of QDA is increased from $(63.72 \%, 66.82 \%$, $99.97 \%$ and $79.07 \%)$ to $(82.69 \%, 84.84 \%, 96.68 \%$ and $83.12 \%)$. We can see that HAFS is effective and can improve the performance of the method for different methods. The 
Table 6 Performance of HAFS achieved by SVM, KNN, GaussianNB and QDA by using and not using HAFS

\begin{tabular}{|c|c|c|c|c|c|c|}
\hline Method & $\alpha$ & Label & Precision (\%) & Recall (\%) & Accuracy (\%) & F-measure (\%) \\
\hline \multirow[t]{4}{*}{ SVM } & & 1 & 76.34 & 99.42 & 82.3 & 86.37 \\
\hline & & 2 & 99.48 & 62.07 & 92.31 & 76.45 \\
\hline & & 3 & 100.0 & 57.75 & 98.04 & 73.21 \\
\hline & & 4 & 96.84 & 58.2 & 91.75 & 72.71 \\
\hline \multirow[t]{4}{*}{ HAFS-SVM } & 0.1 & 1 & 90.08 & 95.03 & 91.3 & 92.49 \\
\hline & & 2 & 91.64 & 87.03 & 95.8 & 89.28 \\
\hline & & 3 & 99.1 & 77.46 & 98.92 & 86.96 \\
\hline & & 4 & 84.98 & 80.14 & 93.58 & 82.49 \\
\hline \multirow[t]{4}{*}{ KNN } & & 1 & 88.04 & 86.32 & 85.66 & 87.17 \\
\hline & & 2 & 83.09 & 83.23 & 93.19 & 83.16 \\
\hline & & 3 & 78.05 & 86.49 & 98.17 & 82.05 \\
\hline & & 4 & 65.98 & 67.96 & 87.58 & 66.96 \\
\hline \multirow[t]{4}{*}{ HAFS-KNN } & 0.5 & 1 & 89.01 & 87.01 & 86.6 & 88.0 \\
\hline & & 2 & 83.17 & 84.52 & 93.42 & 83.84 \\
\hline & & 3 & 80.77 & 85.14 & 98.31 & 82.89 \\
\hline & & 4 & 66.89 & 69.37 & 87.97 & 68.11 \\
\hline \multirow[t]{4}{*}{ GaussianNB } & & 1 & 88.57 & 59.6 & 72.88 & 71.25 \\
\hline & & 2 & 42.62 & 62.72 & 75.52 & 50.75 \\
\hline & & 3 & 14.64 & 86.62 & 76.01 & 25.05 \\
\hline & & 4 & 37.82 & 10.19 & 79.89 & 16.05 \\
\hline \multirow[t]{4}{*}{ HAFS-GaussianNB } & 0.1 & 1 & 81.77 & 77.8 & 77.71 & 79.74 \\
\hline & & 2 & 46.21 & 51.38 & 78.19 & 48.66 \\
\hline & & 3 & 34.96 & 55.63 & 93.16 & 42.93 \\
\hline & & 4 & 46.67 & 41.11 & 80.02 & 43.71 \\
\hline \multirow[t]{4}{*}{ QDA } & & 1 & 95.14 & 36.67 & 63.72 & 52.94 \\
\hline & & 2 & 39.1 & 97.27 & 66.82 & 55.78 \\
\hline & & 3 & 100.0 & 99.3 & 99.97 & 99.65 \\
\hline & & 4 & 43.38 & 48.66 & 79.07 & 45.87 \\
\hline \multirow[t]{4}{*}{ HAFS-QDA } & 0.3 & 1 & 86.07 & 82.19 & 82.69 & 84.09 \\
\hline & & 2 & 60.85 & 82.88 & 84.84 & 70.17 \\
\hline & & 3 & 58.93 & 92.96 & 96.68 & 72.13 \\
\hline & & 4 & 56.51 & 31.84 & 83.12 & 40.73 \\
\hline
\end{tabular}

reason may be that HAFS can select a small number of irrelevant features from a large number of features, thereby avoiding the phenomenon of over-fitting. And we can see that for different models, the best $\alpha$ is different, for example, for SVM, the best $\alpha$ is $0.1, \mathrm{KNN}$ is 0.5 , GaussianNB is 0.1 , and QDA is 0.3 . The possible reason for the difference is that the principle of the classifier is not the same.

\section{Discussion}

For the detection of patients with new coronary pneumonia, the use of machine learning to make judgments is the current mainstream method [10-13]. This is because of the amount of data required for machine learning is small and the characteristics of the lesion can be characterized by extracting its features from the patient's CT image, the model can be fitted with less data. At present, the subtype classification of COVID-19 lesions, which is similar 
to the classic classification task, has only been studied by very few people. Therefore, similar to most previous work, in this paper, we also use machine learning methods to classify COVID-19 lesion subtypes.

Compared with the previous method, HAFS-RF has achieved excellent performance. There are two main reasons: (1) for the data set, we use the ADASYN algorithm to enhance the data of different types of samples to alleviate the imbalance of the data in the training set. (2) For each lesion, our group uses more features, including three-dimensional features such as GLCM and LDP, which can better represent the information of the lesion. (3) For the training model, we designed an HAFS feature selection algorithm, which first uses the GA algorithm performs a feature selection, and then uses the F-test [32], MINE, RFE [34], and Lasso [35] algorithm to score the features, and selects the head features with higher comprehensive scores, and discards the tail features. Doing so can filter out features with low influence, avoid feature redundancy, and reduce the amount of calculation.

Table 5 shows the performance scores obtained after applying HAFS and other classic feature selection algorithms to our model. It can be seen that the scores obtained by HAFS are the best. It can be seen from Table 1 that the model we used HAFS-RF is better than the traditional RF model, and even scored higher than other models such as SVM.

However, the problem of data imbalance between different subtypes is still difficult to solve, and more CT data will be collected in the next step. In addition, with the increase of COVID-19 image data, deep learning can increasingly exert its superior performance. We will try to use CNN to implement a point-to-point COVID-19 subtype discrimination model in the future. In addition, in our model, the weighting factor $\alpha$ is generally a specific value (i.e., $[0,1.0])$. In the future, we will change it to a learnable parameter, so that the performance of the model may be further improved. Finally, it is worth mentioning that the HAFS feature selection method we proposed can be used as a basic method for other machine learning models.

\section{Conclusion}

The most four typical lesion subtypes of COVID-19 are discussed and a computer-aided diagnosis approach of lesion subtype is proposed in this paper. Then the three-dimensional texture descriptors are applied on the volume data of lesions as well as shape and first-order features. The massive feature data are selected by hybrid adaptive selection algorithm and a classification model is trained at the same time. Extensive experiments on clinical realworld datasets demonstrate the effectiveness of the proposed model of HAFS-RF. Moreover, we show the capability of the proposed model for the high dimension feature data with serious imbalance problem. The results show that the 3D radiomics features chosen by HAFS algorithm can better express the advanced information of the lesion data. The classification model obtains a good performance and is compared the models of COVID-19 in the state of art, which can help clinicians more accurately identify the subtypes of COVID19 lesions and provide help for further research.

\section{Materials and methods}

In this study, four lesion subtypes are studied, namely ground-glass opacity (GGO, referred as label 1), cord (referred as label 2), solid (referred as label 3), and subsolid (referred as label 4) [22]. The CT images of the prepared dataset in this paper are shown 


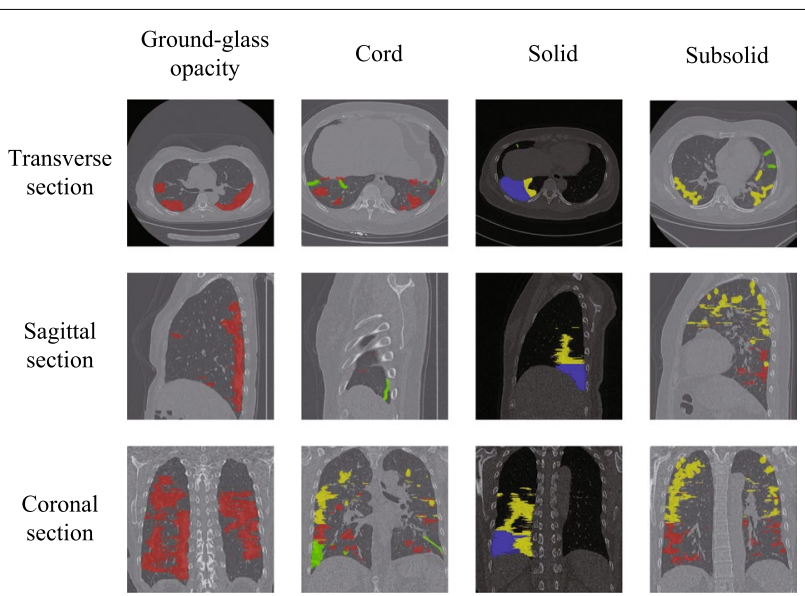

Fig. 3 Typical four lesion subtypes in CT images of COVID-19. The labels and regions are given by medical experts. The red area represents ground-glass opacity, the green area represents cord, the blue area represents solid and the yellow area represents subsolid

Table 7 Samples of lesion from prepared dataset COVID-19

\begin{tabular}{|c|c|c|c|c|c|c|}
\hline Label & Num & Statistics & $X$-length & $Y$-length & Z-length & Volume \\
\hline \multirow[t]{3}{*}{1} & 2637 & Max & 247.0 & 312.0 & 399.0 & 20148480.0 \\
\hline & & Mean & 46.78 & 49.2 & 24.84 & 395826.08 \\
\hline & & Std & 42.2 & 50.68 & 39.63 & 1596465.23 \\
\hline \multirow[t]{3}{*}{2} & 519 & Max & 186.0 & 205.0 & 310.0 & 5142630.0 \\
\hline & & Mean & 43.38 & 41.28 & 24.86 & 124041.76 \\
\hline & & Std & 31.4 & 27.92 & 28.17 & 409901.04 \\
\hline \multirow[t]{3}{*}{3} & 103 & Max & 217.0 & 301.0 & 223.0 & 5878530.0 \\
\hline & & Mean & 40.46 & 37.9 & 21.53 & 210307.5 \\
\hline & & Std & 46.06 & 45.99 & 26.49 & 752977.45 \\
\hline \multirow[t]{3}{*}{4} & 475 & Max & 204.0 & 283.0 & 378.0 & 16873920.0 \\
\hline & & Mean & 61.9 & 63.26 & 38.79 & 722428.14 \\
\hline & & Std & 50.65 & 57.84 & 53.43 & 1979445.47 \\
\hline
\end{tabular}

in Fig. 3, which are presented in transverse, sagittal and coronal plane, respectively, and the lesions are annotated by two radiologists with ITK-SNAP software.

The numbers of the four types of lesions are 2637, 519, 103 and 475, respectively, and the total is 3734 in original dataset. Table 7 shows the summary of the prepared dataset that are maximum, minimum and standard variance values of the sizes of three directions and volumes for each lesions subtypes. The subtype of ground-glass opacity hosts the majority of COVID-19 which shows imbalance data problem. The subsolid subtype is the largest in size of lesions while the cord subtype is the smallest one.

Based on this data set, the flowchart of the algorithm is shown in Fig. 4 and the architecture of the algorithm is shown in Fig. 5 which includes four steps. We firstly introduce the algorithm of lesions extraction and data augmentation used in this study. Then, the feature extraction process for the 2D and 3D features are discussed. The implementation details are presented subsequently. Finally, we describe the random forest model in the forth step. 

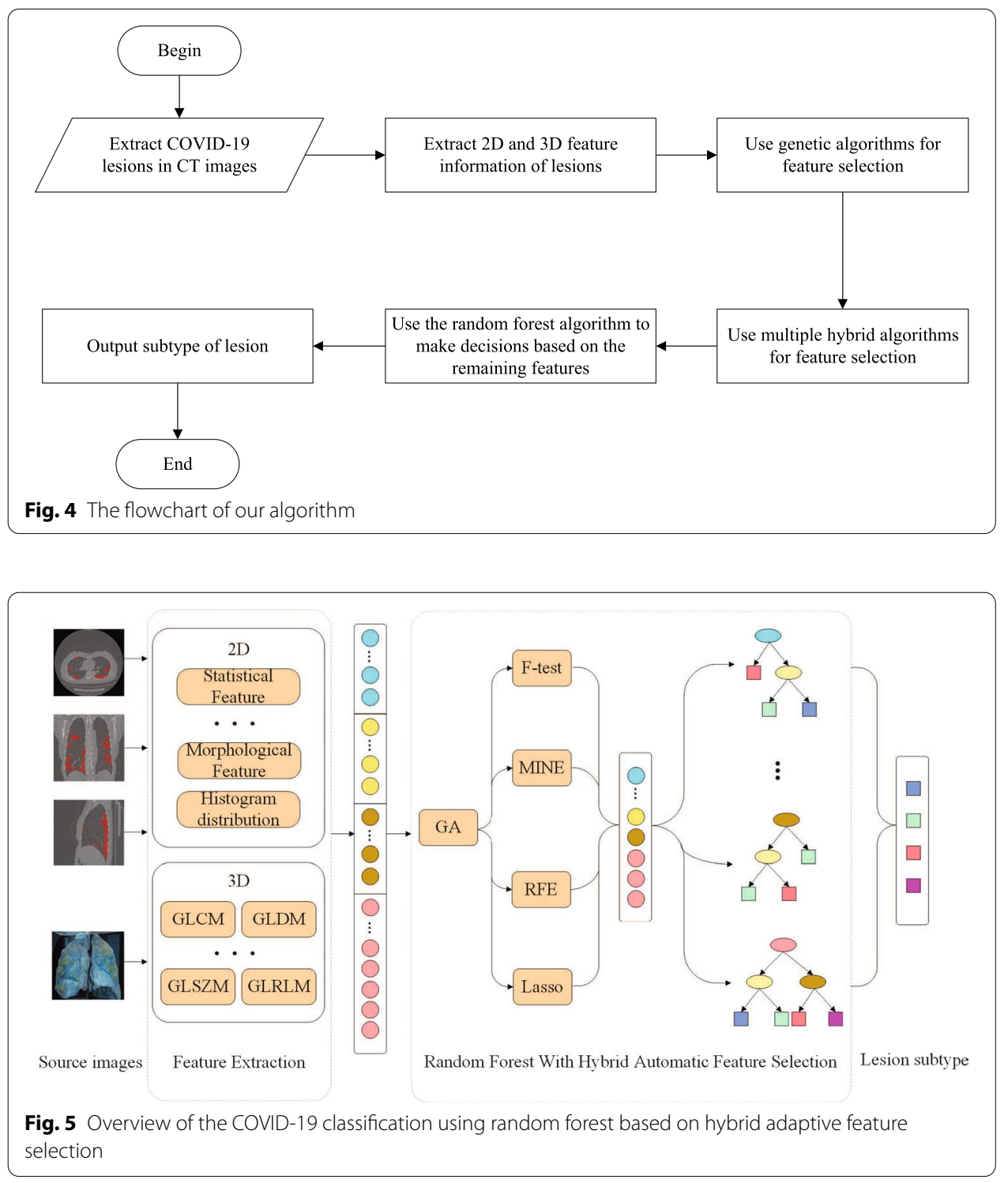

\section{Lesions data extraction and augmentation}

VB-Net is to predict and segment the image of the unknown lesion location. It combines $\mathrm{V}-\mathrm{Net}$ and bottleneck layers to reduce and combine redundant information [23]. However, all the $3 \mathrm{D}$ volume data used in this experiment has the mask position of the corresponding lesion from annotations by the radiologists. Therefore, the VB-Net or V-Net will cause an error in extracting lesions. So we firstly use a breadth-first traversal (BFS) based lesion extraction algorithm to extract lesions which is mostly used in graph structure data. It should be noted that the lesion locations and subtypes are labeled by two radiologists that ensure the accuracy of evaluation data.

The BFS-based lesion extraction algorithm is shown as Algorithm 1. We traverse the mask array, which is created by radiologists, in the entire 3D volume data. When traversing the pixels with a lesion mark, this paper uses the BFS to extract the lesion range from the case with that mask [24]. The input is generally a point in the graph, 
then use the point to initialize a queue. The main idea of the algorithm is to take out a point from it each time, and then for this point, all nearby points that meet the requirements are enqueued, and then the above process is repeated until the queue is empty.

Input: 3D coordinates of a point, $A$; Mask of the lesion, Mask; Array of

Output: Range mark, mark; Length of the search range, $L$.

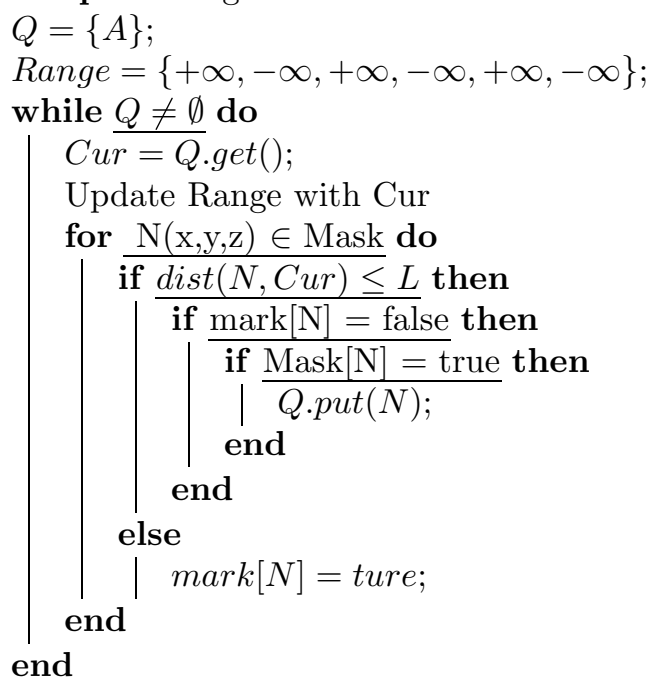

Algorithm 1: The lesion extraction algorithm.

Because the setting of the search range $L$ in the algorithm, the algorithm can well avoid the discontinuity of the lesion data in a certain dimension caused by inaccurate data labeling. And we set a global mark in the algorithm, all traversed points will be recorded. There are two advantages to this:

1. In the current BFS process, the marked points will not be enqueued, which can avoid double calculation;

2. In the global traversal of the mask data, the marked points will not call the BFS algorithm repeatedly. This can ensure that each time the BFS algorithm only generates one lesion and returns its $3 \mathrm{D}$ ranges.

As we all know, the data imbalance problem will lead to a decrease in the accuracy of multi-classification. The four different subtypes of lesions number are 2637, 519, 103 and 475. Obviously they are unbalanced, and the number of label 1 lesions is far greater than label 3, which can leads to insufficient fitting ability of the classifier to label 3 samples. The unbalance characteristic data can shift the decision boundary of the classifier, and affect the final classification effect. Therefore, we have adopted a data enhancement method based on ADASYN proposed by He [25] to reduce its impact on classification accuracy. This paper adopts a data enhancement method based ADASYN to increase the number of label 2, 3 and 4. The method is briefly introduced in Algorithm 2. 
Input: Dataset train $_{\text {. }}$

Output: The synthetic data $x_{\text {syn }}$

The number of majority class is defined as: $n_{l}$;

The number of minority class is defined as: $n_{s}$;

Calculate the degree of class imbalance: Degree imbalance $=n_{s} / n_{l}$;

if Degree $_{\text {imbalance }}<$ Degree $_{\text {threshold }}$ then

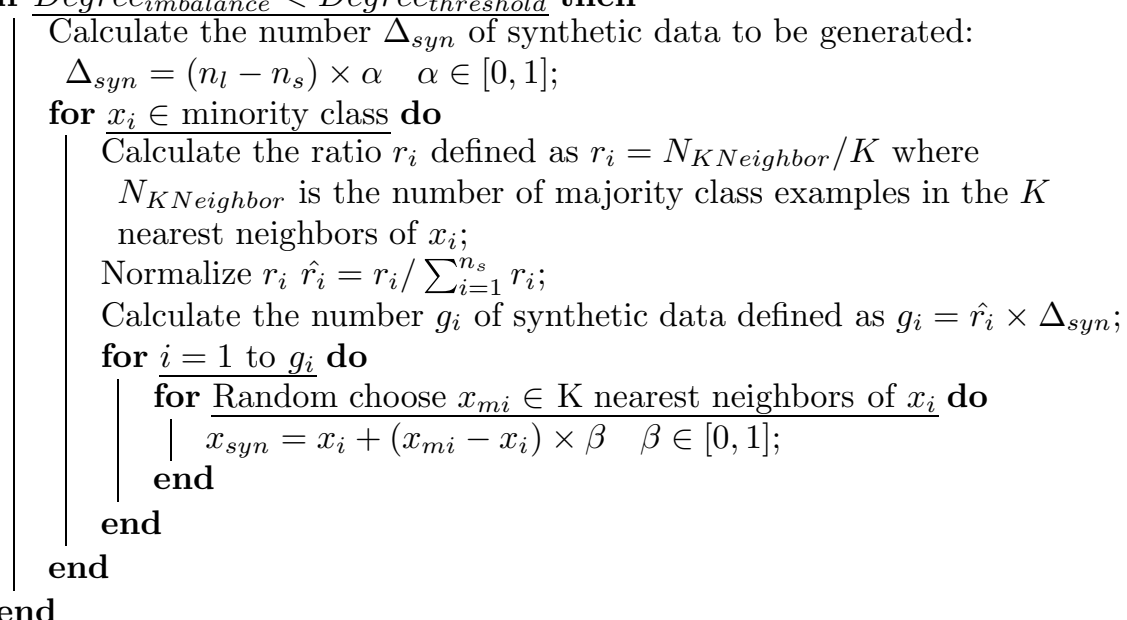

Algorithm 2: The algorithm of data enhancement based on ADASYN in lesion subtypes unbalance improvement process.

The algorithm increases the number of four subtype lesions from $(2637,519,103$ and 475$)$ to $(2637,1098,386$ and 976) which is evaluated that the data augmentation can effectively improve the classification performance evaluated by the experiments in "Results" section .

\section{Three-dimensional feature extraction}

The most of the current medical imaging research on COVID-19 are mainly based on $\mathrm{X}$-rays images or ignoring the characteristics of $\mathrm{CT}$ planar images. Furthermore, 3D features are also seldom considered in the research. Therefore, this paper extracted the 2D features of some certain layers and more 3D features of the CT data to better characterize the lesion information.

The existing methods are mainly based on extracting 2D features from CT images. This ignores that the COVID-19 lesion is a kind of volume data. Therefore, in the process of feature extraction, the connection between layers is ignored, and some hidden features are lost.

In order to improve the accuracy of classification, we extracted multiple types of features of the lesion that include 2D and 3D features and are shown in detail as following:

1. Infected lesions number: The stage of the COVID-19 affects the number of lesions and also affects the distribution of different types. Therefore, we add the total number of lesions in the same patient.

2. Shape features: Some cord-type lesions are significantly different from other lesions in shape, so we extracted three-dimensional shape features from the lesions in order to improve the accuracy of multi-classification. 
3. First-order features: The first-order features provide information related to the graylevel distribution of the image. We first obtain the middle layer and the layer with the largest lesion area of CT images in three directions, and extract 14 two-dimensional manual features from them, including mean, var, max, skewness, kurtosis, area, compact, rough, contrast, dissimilarity, homogeneity, energy, correlation, ASM (active shape model). Then we also extract the three-dimensional features and hybrid them into the total features.

4. Second-order features: Second-order features give more information about the relative positions of the various gray levels within the lesion image.

a. The gray-level co-occurrence matrix (GLCM) [26]: The GLCM is a statistical method of analyzing texture that considers the spatial relationship of pixels. The $(i, j)$ th element of GLCM $P(i, j \mid \delta, \theta)$ describes the number of times the combination of levels $i$ and $j$ occur in two pixels in the image, that are separated by a distance of $\delta$ pixels along angle $\theta$. The 3D-GLCM considers 13 directions and need to be calculated separately and finally averaged.

b. The gray-level run length matrix (GLRLM) [27]: The GLRLM quantifies graylevel runs, which are defined as the length in number of pixels of the same graylevel value. The $(i, j)$ th element of the $\operatorname{GLRLM} P(i, j \mid \delta, \theta)$ represents the number of runs with gray level $i$ and length $j$ occur in the image along angle $\theta$. Similar to 3D-GLCM, 3D-GLRLM also needs to be calculated separately for 13 directions.

c. The gray-level size zone matrix (GLSZM) [28]: In the $\operatorname{GLSZM~} P(i, j)$, the $(i, j)$ th element represents the number of zones with gray level $i$ and size $j$ appear in the image. A zone is defined as the number of connected voxels that share the same gray-level intensity. Contrary to GLCM and GLRLM, the 3D-GLSZM is rotation independent, with only one matrix calculated for all directions.

d. Gray-level dependence matrix (GLDM) [29]: The GLDM quantifies gray-level dependencies in the image. The $(i, j)$ th element in GLDM $P(i, j \mid \alpha)$ equals the number of times a voxel with gray level $i$ with $j$ dependent voxels in its neighborhood appears in an image. A neighboring voxel with gray level $j$ is considered dependent on center voxel with gray level $i$ if $|i-j| \leq \alpha$. Similar to 3D-GLRLM, the 3D-GLDM is also rotation independent.

We introduced some 3D features from GLCM, GLSZM, GLRLM, GLDM, and the numbers are $22,16,16,14$, respectively. The detailed information of features can be found in the Pyradiomics [30] document on [31]. These features correspond to the GLCM(1-15 16-21 23-24), GLRLM(1-16), GLSZM(1-16), and $\operatorname{GLDM}(1-14)$.

In addition, we also extracted 4 features that are width, height, length and volume of the 3D lesion from the bounding box of COVID-19 lesion. In summary, a total of 189 features are used in our study. 


\section{Hybrid adaptive feature selection method}

As described in "Three-dimensional feature extraction" section, we extract specific features from 3D lesion data. However, some irrelevant features can easily cause over-fitting of the model, which will reduce the accuracy of the test set. Therefore, before training the model, it is necessary to perform feature selection processing to reduce the influence of the redundant feature.

There are three kinds of algorithms in the existing feature selection process, namely filters, wrappers, and embedded. Although these methods have their advantages, they all have one obvious disadvantage that the number of features in the subset after feature selection cannot be determined. So at this stage, we proposed a Hybrid Adaptive Feature Selection (HAFS) algorithm. It can not only solve the problem of the uncertain number of features in the subset, but also integrate the advantages of various traditional methods.

HAFS method is divided into two stages. In the first stage, the feature set $F=\left\{f_{1}, f_{2} \ldots f_{189}\right\}$ is used as input, and the genetic algorithm (GA) is used to accurately select features. First, a population of $q$ chromosomes will be initialized, and each chromosome is a binary set of length $n U=\left\{U_{1}, U_{2} \ldots U_{189}\right\}$, each value of $U_{i}$ is 1 or 0 , if $U_{i}=1$, it means $f_{i}$ is selected, otherwise, $f_{i}$ is not selected. Next, $p$ iteration will be performed. Before each iteration, individual fitness is evaluated for each chromosome. Then, according to the fitness, the chromosomes in the population are calculated with different probabilities of three genetic operators that are selection, crossover, and mutation. At the end of the iteration, the feature subset $F_{\mathrm{GA}}=\left\{f_{\mathrm{GA} 1}, f_{\mathrm{GA} 2} \ldots f_{\mathrm{GA} m}\right\}$ is determined according to the value of each bit according to the chromosome $U_{\max }$ with the highest fitness. A schematic illustration of the first stage is shown in Fig. 6.

In the second stage, in order to integrate the advantages of multiple feature selection methods, we use $F_{\mathrm{GA}}$ as input, and take two filters method that are $F$-test [32] and maximal information coefficient (MIC) [33], one wrappers method that is recursive feature elimination (RFE) [34], and one embedded method that is L1 regularized linear regression model(Lasso) [35] into data preprocessing methods to score features and sort them in ascending order, the results are presented as $F_{1}, F_{2}, F_{3}$ and $F_{4}$ :

$$
\begin{aligned}
& F_{1}=F-\operatorname{test}\left(F_{\mathrm{GA}}\right)=\left\{f_{F 1}, f_{F 2} \ldots f_{F m}\right\}, \\
& F_{2}=\operatorname{MIC}\left(F_{\mathrm{GA}}\right)=\left\{f_{M 1}, f_{M 2} \ldots f_{M m}\right\}, \\
& F_{3}=\operatorname{RFE}\left(F_{\mathrm{GA}}\right)=\left\{f_{R 1}, f_{R 2} \ldots f_{R m}\right\}, \\
& F_{4}=\operatorname{Lasso}\left(F_{\mathrm{GA}}\right)=\left\{f_{L 1}, f_{L 2} \ldots f_{L m}\right\} .
\end{aligned}
$$

So we score each $f_{\mathrm{GA}}$ in $F_{\mathrm{GA}}$ according to $F_{1}, F_{2}, F_{3}$ and $F_{4}$. According to the $S\left(f_{\mathrm{GA}}\right)$ score, $F_{\mathrm{GA}}$ is sorted in ascending order. In this way, we can decide which features to keep. The scoring standard, $S\left(f_{\mathrm{GA}}\right)$, is:

$$
S\left(f_{\mathrm{GA}}\right)=\frac{\sum_{i=1}^{4} \operatorname{index}\left(F_{i}, f_{\mathrm{GA}}\right)}{4} .
$$




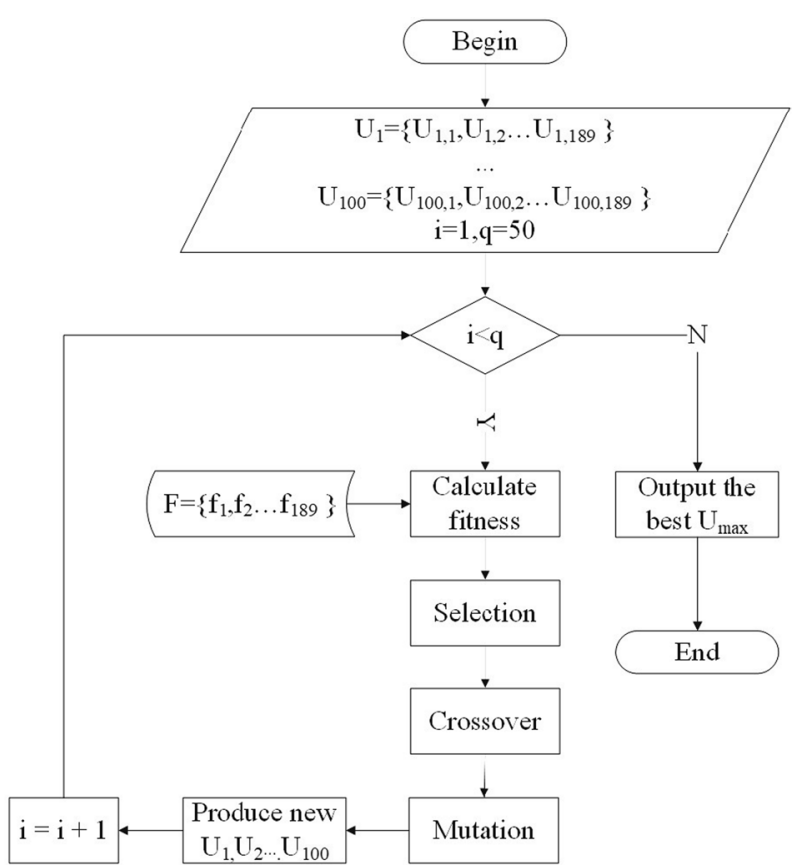

Fig. 6 A schematic illustration of first stage of HAFS

On the other hand, in order to further prevent feature over-fitting after GA selection, some features of $F_{\mathrm{GA}}$ are selected and a parameter $\alpha$ is set as the ratio of the number of selected features. The final result of feature selection $F_{\mathrm{HAFS}}$ is:

$$
F_{\mathrm{HAFS}}=\alpha F_{\mathrm{GA}} .
$$

\section{Random forest-based classification model}

In the classification stage, the classifier used in this paper is random forest (RF) model. The main idea is: select a subset $S_{\mathrm{b}}$ from all sample set $S$ through randomly selecting sample features and sampling. Then a classification and regression tree is established for $S_{\mathrm{b}}$. The classification and regression tree uses the Gini coefficient as the criterion [36]. In this paper, the above process was repeated 1000 times to construct 1000 CART trees to construct a random forest. The random forest-based classification model is shown in Algorithm 3. 
Input: Samples $S$; Features of the lesion $F$; Number of iterations $m$.

Output: Type of lesion predicted, $P$.

$\mathrm{j}=1$;

$\mathrm{m}=1000$;

while $\mathrm{j} \leq \mathrm{m}$ do

Take $S_{b}$ samples with Bootstrap from $S$.

Randomly select $F_{s}$ features from $F_{H A F S}$.

Build a CART tree by $S_{b}$ samples and $F_{s}$ features.

$j=j+1$;

end

For each new sample. Generate $m$ results through the CART trees.

Determine the type of lesion by the principle of minority obeying the majority $P$.

Algorithm 3: Random Forest algorithm for COVID-19 Classification.

\section{Experimental settings}

Since COVID-19 is a new disease, there are few public data sets of CT images with annotations suitable for this study. Therefore, we extracted lesions from 319 cases of COVID19 pneumonia patients provided by Neusoft Medical to construct a dataset. All patients received a thin-slice CT scan of the chest by Neusoft 256 slice CT.

The final subsets of features are evaluated by RF classifications associated with tenfold cross-validations. Precision, recall, accuracy and $F$-measure are used to compare the estimated and known labels according to the following expressions:

$$
\begin{aligned}
& \text { Precision }=\frac{\mathrm{TP}}{\mathrm{TP}+\mathrm{FP}}, \\
& \text { Recall }=\frac{\mathrm{TP}}{\mathrm{TP}+\mathrm{FN}}, \\
& \text { Accuracy }=\frac{\mathrm{TP}+\mathrm{TN}}{\mathrm{TP}+\mathrm{TN}+\mathrm{FP}+\mathrm{FN}}, \\
& F \text {-measure }=\frac{2 * \text { Precision } * \text { Recall }}{\text { Precision }+ \text { Recall }},
\end{aligned}
$$

where TP, TN, FP and FN in Eqs. 7-10 represent true positive, true negative, false positive, and false negative, respectively.

In our experiments, we compare our model with the following widely adopted machine learning methods. These models are relatively classic and commonly used models in the field of machine learning.

1. Logistic regression (LR)

2. Support vector machine (SVM) (use a radial basis function kernel with default parameters.) 


\author{
3. K-neighbors classifier (KNN) (set $k=20$ through cross-validation) \\ 4. Decision tree classifier (DT) \\ 5. GaussianNB \\ 6. Quadratic discriminant analysis (QDA) \\ 7. Random forest (RF)
}

\title{
Abbreviations
}

CT: Computed tomography; RT-PCR: Reverse transcription-polymerase chain reaction; GGO: Ground-glass opacity; GLCM: Gray-level co-occurrence matrix; LDP: Local directional pattern; GLRLM: Gray-level run length matrix; GLSZM: Gray-level size zone matrix; IRF: Iterative ReliefF; IAVP: Influenza-A viral pneumonia; BFS: Breadth-first traversal; GLDM: Gray-level dependence matrix; GA: Genetic algorithm; MIC: Maximal information coefficient; RFE: Recursive feature elimination; RF: Random forest; KNN: K-Neighbors classifier; DT: Decision tree; QDA: Quadratic discriminant analysis.

\section{Acknowledgements}

Not applicable.

\section{Authors' contributions}

LW proposed experimental ideas, evaluated experimental data, and drafted manuscripts. CYY and KY designed experimental procedures collected data, and assisted in manuscript writing. CYB, HF and YML proposed the overall structure of the article and supplemented the experimental chart. WDX revised the manuscript and evaluated the data. All authors read and approved the final manuscript.

\section{Funding}

This work was supported by National Natural Science Foundation of China (No. U1708261), Fundamental Research Funds for the Central Universities (N2016006) and Shenyang Medical Imaging Processing Engineering Technology Research Center (17-134-8-00)

\section{Data availability statement}

The patient population data used to support the findings of this study have not been made available because the data are supplied by Neusoft under license and so cannot be made freely available. Requests for access to these data should be made to the corresponding author.

\section{Declarations}

\section{Ethics approval and consent to participate}

This article does not contain any studies with human participants or animals performed by any of the authors.

\section{Consent for publication}

Not applicable.

\section{Competing interests}

There are no conflicts of interest declared.

\section{Author details}

${ }^{1}$ Key Laboratory of Intelligent Computing in Medical Image (MIIC), Northeastern University, Ministry of Education, Shenyang, China. ${ }^{2}$ School of Computer Science and Engineering, Northeastern University, Shenyang, China. ${ }^{3}$ Biomedical and Information Engineering School, Northeastern University, Shenyang, China. ${ }^{4}$ Neusoft Medical System Co., Ltd., Shenyang, Liaoning, China.

Received: 17 May 2021 Accepted: 19 November 2021

Published online: 05 December 2021

\section{References}

1. Wang C, Horby PW, Hayden FG, Gao GF. A novel coronavirus outbreak of global health concern. Lancet. 2020;395(10223):470-3.

2. Lu H, Stratton CW, Tang Y-W. Outbreak of pneumonia of unknown etiology in Wuhan, China: the mystery and the miracle. J Med Virol. 2020;92(4):401-2.

3. Johns Hopkins University: Coronavirus Disease (COVID-19) outbreak situation. 2021. https://coronavirus.jhu.edu/.

4. Woloshin S, Patel N, Kesselheim AS. False negative tests for sars-cov-2 infection-challenges and implications. N Engl J Med. 2020;383(6):e38.

5. Li X, Zeng W, Li X, Chen H, Shi L, Li X, Xiang H, Cao Y, Chen H, Liu C, et al. Ct imaging changes of corona virus disease 2019 (covid-19): a multi-center study in southwest China. J Transl Med. 2020;18:1-8.

6. Peng S, Huang L, Zhao B, Zhou S, Braithwaite I, Zhang N, Fu X. Clinical course of coronavirus disease 2019 in 11 patients after thoracic surgery and challenges in diagnosis. J Thorac Cardiovasc Surg. 2020;160(2):585-92. 
7. Zhang S-Y, Lian J-S, Hu J-H, Zhang X-L, Lu Y-F, Cai H, Gu J-Q, Ye C-Y, Jin C-L, Yu G-D, et al. Clinical characteristics of different subtypes and risk factors for the severity of illness in patients with covid-19 in Zhejiang, China. Infect Dis Poverty. 2020;9(1):1-10,

8. Zhao D, Yao F, Wang L, Zheng L, Gao Y, Ye J, Guo F, Zhao H, Gao R. A comparative study on the clinical features of covid-19 pneumonia to other pneumonias. Clin Infect Dis. 2020;71(15):756-61.

9. Zhao W, Zhong Z, Xie X, Yu Q, Liu J. Relation between chest ct findings and clinical conditions of coronavirus disease (covid-19) pneumonia: a multicenter study. Am J Roentgenol. 2020;214(5):1072-7.

10. Shi W, Peng X, Liu T, Cheng Z, Lu H, Yang S, Zhang J, Li F, Wang M, Zhang X, et al. Deep learning-based quantitative computed tomography model in predicting the severity of COVID-19: A retrospective study in 196 patients. SSRN Electron J. 2020. https://doi.org/10.2139/ssrn.3546089.

11. Barstugan M, Ozkaya U, Ozturk S. Coronavirus (covid-19) classification using ct images by machine learning methods. 2020. arXiv preprint arXiv:2003.09424.

12. Ozkaya U, Ozturk S, Barstugan M. Coronavirus (covid-19) classification using deep features fusion and ranking technique. 2020. arXiv preprint arXiv:2004.03698.

13. Elaziz MA, Hosny KM, Salah A, Darwish MM, Lu S, Sahlol AT. New machine learning method for image-based diagnosis of covid-19. PLoS ONE. 2020;15(6):0235187.

14. Tuncer T, Dogan S, Ozyurt F. An automated residual exemplar local binary pattern and iterative relieff based corona detection method using lung x-ray image. Chemom Intell Lab Syst. 2020;203:104054.

15. Zhou T, Canu S, Ruan S. An automatic covid-19 ct segmentation network using spatial and channel attention mechanism. 2020. arXiv preprint arXiv:2004.06673.

16. Khan Al, Shah JL, Bhat MM. Coronet: a deep neural network for detection and diagnosis of covid-19 from chest $x$-ray images. Comput Methods Progr Biomed. 2020;196:105581.

17. Afshar P, Heidarian S, Naderkhani F, Oikonomou A, Plataniotis KN, Mohammadi A. Covid-caps: a capsule networkbased framework for identification of covid-19 cases from x-ray images. 2020. arXiv preprint arXiv:2004.02696.

18. Khalifa NEM, Taha MHN, Hassanien AE, Elghamrawy S. Detection of coronavirus (covid-19) associated pneumonia based on generative adversarial networks and a fine-tuned deep transfer learning model using chest $\mathrm{x}$-ray dataset. 2020. arXiv preprint arXiv:2004.01184.

19. Minaee S, Kafieh R, Sonka M, Yazdani S, Soufi GJ. Deep-covid: predicting covid-19 from chest x-ray images using deep transfer learning. 2020. arXiv preprint arXiv:2004.09363.

20. He K, Zhao W, Xie X, Ji W, Liu M, Tang Z, Shi F, Gao Y, Liu J, Zhang J, et al. Synergistic learning of lung lobe segmentation and hierarchical multi-instance classification for automated severity assessment of covid-19 in ct images. 2020. arXiv preprint arXiv:2005.03832.

21. Xu X, Jiang X, Ma C, Du P, Li X, Lv S, Yu L, Ni Q, Chen Y, Su J, et al. A deep learning system to screen novel coronavirus disease 2019 pneumonia. Engineering. 2020;6(10):1122-9.

22. Kim H, Park CM, Koh JM, Lee SM, Goo JM. Pulmonary subsolid nodules: what radiologists need to know about the imaging features and management strategy. Diagn Interv Radiol. 2014;20(1):47.

23. Milletari F, Navab N, Ahmadi S-A. V-net: fully convolutional neural networks for volumetric medical image segmentation. In: 2016 fourth international conference on 3D vision (3DV), IEEE. 2016. p. 565-71.

24. Beamer S, Asanovic K, Patterson D. Direction-optimizing breadth-first search. In: SC'12: proceedings of the international conference on high performance computing, networking, storage and analysis, IEEE. 2012. p. 1-10.

25. He H, Bai Y, Garcia EA, Li S. Adasyn: adaptive synthetic sampling approach for imbalanced learning. In: 2008 IEEE international joint conference on neural networks (IEEE world congress on computational intelligence), IEEE. 2008. p. 1322-8.

26. Haralick RM, Shanmugam K, Dinstein IH. Textural features for image classification. IEEE Trans Syst Man Cybern. 1973;6:610-21.

27. Mohanty AK, Beberta S, Lenka SK. Classifying benign and malignant mass using glcm and glrlm based texture features from mammogram. Int J Eng Res Appl. 2011;1 (3):687-93.

28. Thibault G, Fertil B, Navarro C, Pereira S, Cau P, Levy N, Sequeira J, Mari J-L. Shape and texture indexes application to cell nuclei classification. Int J Pattern Recogn Artif Intell. 2013;27(01):1357002.

29. Sun C, Wee WG. Neighboring gray level dependence matrix for texture classification. Comput Vis Graph Image Process. 1983:23(3):341-52.

30. Van Griethuysen JJ, Fedorov A, Parmar C, Hosny A, Aucoin N, Narayan V, Beets-Tan RG, Fillion-Robin J-C, Pieper S, Aerts HJ. Computational radiomics system to decode the radiographic phenotype. Cancer Res. 2017;77(21):104-7.

31. van Griethuysen JJM, Fedorov A, Parmar C, Hosny A, Aucoin N, Narayan V, Beets-Tan RGH, Fillon-Robin JC, Pieper S, Aerts HJWL. Pyradiomics documentation. 2017. https://pyradiomics.readthedocs.io.

32. Elssied NOF, Ibrahim O, Osman AH. A novel feature selection based on one-way anova f-test for e-mail spam classification. Res J Appl Sci Eng Technol. 2014;7(3):625-38.

33. Lin C, Miller T, Dligach D, Plenge R, Karlson E, Savova G. Maximal information coefficient for feature selection for clinical document classification. In: ICML workshop on machine learning for clinical data. Edingburgh, UK; 2012.

34. Yan K, Zhang D. Feature selection and analysis on correlated gas sensor data with recursive feature elimination. Sens Actuators B Chem. 2015;212:353-63.

35. Fonti V, Belitser E. Feature selection using lasso. VU Amst Res Pap Bus Anal. 2017;30:1-25.

36. Menze BH, Kelm BM, Masuch R, Himmelreich U, Bachert P, Petrich W, Hamprecht FA. A comparison of random forest and its gini importance with standard chemometric methods for the feature selection and classification of spectral data. BMC Bioinform. 2009;10(1):213.

\section{Publisher's Note}

Springer Nature remains neutral with regard to jurisdictional claims in published maps and institutional affiliations. 